\title{
SISTEMA DE CONTROL DOMÓTICO UTILIZANDO LA RED ELÉCTRICA COMO MEDIO FÍSICO DE TRANSMISIÓN
}

\author{
Juan Carlos Vesga Ferreira ${ }^{14}$
}

RESUMEN

El sistema de comunicación que hace uso de la red eléctrica como medio físico de transmisión PLC (Power Line Communication), apareció alrededor de los años 50 y había sido considerado durante muchos años como una alternativa para la transmisión de información a bajas de transferencia; sin embargo, en la actualidad esto ya no es así, debido a que esta tecnología ha migrado hacia las redes de datos gracias a los avances en los esquemas de modulación tales como: OFDM (Orthogonal frecuency division multiplexing) y CSMA/CA (Carrier Sense Multiple Access with Collision Avoidance).

En este artículo se presenta un prototipo de un sistema de control asistido por computador, el cual permite disponer de una vivienda o instalación dotada de un Sistema de Control capaz de realizar cualquier función que se le pida utilizando la red eléctrica como medio físico de transmisión. El módulo se programa de manera independiente a través del software de control según su identificación, lo que permite conformar sistemas de control distribuido en forma modular. Además, presenta protección de sobre corrientes, debido a que posee fusibles en caso de sobrecargas de energía a través de alguna de sus salidas. Cada una de las salidas presenta funciones como: interruptor, interruptor programado, DIMMER para control de tensión aplicada a la carga.

La domótica se muestra como toda una promesa que revolucionará muchos hábitos y facilitará las actividades mecánicas, monótonas o triviales, brindando mayor seguridad dentro del hogar. Por el momento, los altos costos de instalación de estos sistemas, hacen que la domótica llegue a un número limitado de usuarios; sin embargo, la tecnología seguirá su natural proceso exponencial de desarrollo, ofreciendo en un par de años más, el acceso a estos sistemas integrales de control casero apto para toda la familia.

Palabras clave: domótica, inmótica, control, red eléctrica, módulo.

\footnotetext{
14 Ingeniero Electrónico, Ingeniero de Sistemas; Especialista en Telecomunicaciones, Especialista en Docencia Universitaria; Msc(c) en Telecomunicaciones. Coordinador Nacional Ingeniería en Telecomunicaciones UNAD, líder del grupo de investigación GIETA, Legal Main Contact Academia CISCO-UNAD, Instructor CISCO CCNA, CCNP-CCAI. Grupo de Investigación: GIETA "Grupo de Investigación en Electrónica, Telecomunicaciones y Audio" (UNAD). Código del Grupo en Colciencias: GIETA: COL0091869, Nombre del semillero: SIETEL "Grupo de investigación en Electrónica y Telecomunicaciones" (UNAD). Línea de investigación: Nuevas Tecnologías en Telecomunicaciones. Red de Investigación: Informática e inclusión.
} 


\begin{abstract}
The system of communication that makes use of the electric net like half a physique of transmission PLC ( Power Line Communication ), appeared around the years 50 and it had been considered during many years like an alternative for the transmission of information transferential casualties; Nevertheless, at present this already is not like that, due to the fact that this technology has migrated towards the networks of information thanks to the advances in the schemes of modulation as: OFDM (Orthogonal frecuency division multiplexing) and CSMA/CA (Multiple Carrier Sense Access with Collision Avoidance).

In this article is present a prototype of a system of control represented by computer, which allows to have a housing or installation provided with a System of Control capable of realizing any function for that he asks him using the electrical network as physical way of transmission.

The module is programmed of independent way through the software of control according to his identification, that allows conforming systems of control distributed in modular form, besides presents protection of on currents, because you possess fuses in case of surcharges of energy through any one of his exits. Each of the exits presents shows like: Breaker, programmed breaker, DIMMER for control of applied tension charges it.

The domotic shows up like all a promising youth that will revolutionize many habits making easy the mechanical, monotonous or trivial activities offering bigger certainty within the home. For the moment the loud costs of installation of these systems, they do that the domótica get to a number limited of users; However, the technology will obey his natural exponential process of development, offering in a couple of years plus access the family to these integral fit for anything systems of homemade control.
\end{abstract}

Key words: domotic, inmotic, control, electric net, module

\title{
INTRODUCCIÓN
}

La red eléctrica es una estructura que hasta el momento ha sido utilizada exclusivamente para el transporte de energía eléctrica. Sin embargo, es posible hacer uso de esta gran red en procesos de comunicación y transmisión de información tales como: telemetría, voz, datos y video; teniendo en cuenta que la red eléctrica no había sido concebida para tal fin. Por tal razón, la tecnología PLC se convierte en un tema de investigación con el objetivo de conocer los parámetros de viabilidad en el país, debido a que algunos aspectos que son descritos en el Código Eléctrico Colombiano (NTC 2050) como los sistemas de puesta a tierra en redes domiciliarias no son implementados acorde con él, lo cual conlleva a que las redes eléctricas presenten altos niveles de ruido y a efectos que se pueden producir en el momento de hacer uso de esta tecnología.

La red eléctrica ha sido considerada últimamente como una alternativa bastante llamativa para la transmisión de datos, su uso en este sentido permite el ahorro de cableado y aprovechamiento de las redes ya construidas en las viviendas actualmente. 
El uso de la red eléctrica como medio físico de transmisión, brinda la posibilidad de establecer procesos de comunicación utilizando infraestructura ya existente, convirtiendo el cableado de distribución doméstico en una red de área local, transformando cada tomacorriente en un punto de acceso a esta red, permitiendo llegar a diferentes rincones donde otros medios de transmisión no llegarían tan fácilmente.

Es por ello que se pensó en el diseño de un prototipo que consiste de un transmisor y un receptor que permitan establecer una comunicación serial entre dos computadores a una velocidad máxima de 600 baudios, utilizando tecnología existente en el mercado y de fácil implementación.

Por otra parte, la domótica es un área de investigación moderna, que persigue el desarrollo de sistemas de automatización de viviendas, abarcando los aspectos relacionados con edificaciones inteligentes, el control de dispositivos domésticos y de seguridad principalmente.

Este trabajo obedece a un desarrollo en domótica acorde con la realidad y necesidades colombianas, haciendo uso de dispositivos y elementos disponibles en el mercado local. El sistema consta de una unidad central que es el PC, una interfaz de comunicación RS232/FSK, un módulo domótico que cuenta con un sistema de comunicación semi-duplex utilizando un protocolo experimental, una etapa de potencia que permite conectar cargas resistivas e inductivas y una terminal adicional para conexión de sensores. Cada módulo es controlado por un microcontrolador PIC16F84 y está en capacidad de realizar funciones especiales tales como el control de la intensidad luminosa de lámparas incandescentes, programación horaria y semanal del encendido y apagado de las diferentes cargas.

Adicionalmente, el manejar la red eléctrica como medio físico de transmisión, utilizando un protocolo de comunicación propietario de tipo experimental, permitirá establecer procesos de comunicación entre el PC y el módulo domótico mejorando las condiciones establecidas por otros protocolos homólogos tales como X10 y CEBus que permiten:

- Alcanzar una velocidad de transmisión de 600 baudios, superando con ello 10 veces la velocidad de comunicación del sistema X10, la cual es de 60 baudios.

- Comunicación semi-duplex

- Envío de datos sin redundancia, es decir, que no sea necesario enviar varias veces la misma trama de comunicación. Tan sólo se envía una vez.

- Verificación de recepción correcta de información por parte del módulo domótico. Situación que no ocurre con los otros sistemas homólogos.

\section{Descripción general del módulo didáctico}

Este sistema de control permite instalar un módulo en cada uno de los recintos a controlar (por ejemplo: un módulo por habitación). Cada módulo está constituido por tres entradas y tres salidas. Cada salida se puede administrar de manera independiente y se configurar de la siguiente forma: una salida para control de luces, otra salida para control de ventiladores o aires acondicionados y otra salida para control de tomacorrientes y electrodomésticos. 
La configuración y control se efectúa mediante un computador, este posee instalado un software que permite comunicarse, controlar y configurar cada una de las salidas y módulos en forma programada y manual según horarios de utilización de los diferentes recintos. Por ejemplo: si se desea que la lámpara de la calle se encienda de lunes a viernes de 6:00 PM a 5:00 AM, este horario se programa en el sistema y el módulo solamente suministrará energía a la lámpara durante ese intervalo de tiempo y en esos días, es decir, que la energía consumida será la necesaria evitando derroches y gastos innecesarios que conllevan a un desperdicio de energía.

Figura 1. Esquema general del Módulo Didáctico en una Vivienda

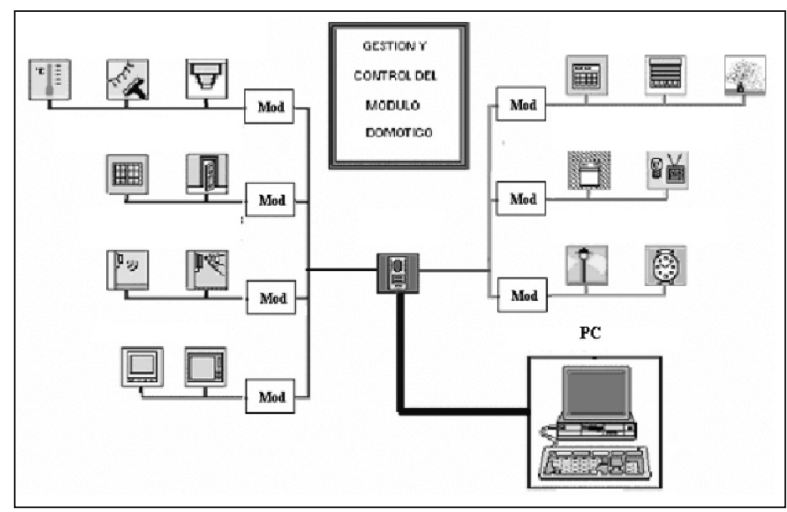

Fuente: el autor

El sistema domótico permite disponer de una vivienda o instalación dotada de un Sistema de Control capaz de realizar cualquier función que le pida. Comunicarse, proteger el establecimiento, controlar encendido y apagado de electrodomésticos, luces, toma corrientes incluyendo una gran variedad de controladores conectados por medio de la red eléctrica.

\section{Descripción general del módulo domótico}

El módulo se encuentra constituido por tres entradas análogas y tres salidas, las cuales pueden establecer una corriente de 20 Amperios cada una. Adicionalmente, presenta dos terminales que se pueden utilizar como terminales para detección, conexión y configuración de sensores de humo, de humedad o de movimiento.

El módulo se programa de manera independiente a través del software de control según su identificación, lo que permite conformar sistemas de control distribuido en forma modular, además, presenta protección de sobre corrientes, debido a que posee fusibles en caso de sobrecargas de energía a través de alguna de sus salidas.

Una de las principales características de éste módulo domótico es que a través del mismo cable, por donde se suministra la energía para alimentación del módulo, se hace el envío y recepción de información al sistema de control. 
Las principales características son:

- Se utilizó Topología BUS para la conexión de los módulos, permitiéndose un máximo de 15 , debido a que se utilizaron 4 bits para la identificación de cada módulo.

- El protocolo de comunicación utilizado para la capa física es el RS232/FSK, el cual permite transmisión de datos serial, con una distancia máxima entre el primero y el último módulo de $100 \mathrm{~m}$ según especificaciones de tensión como norma para el uso de los circuitos integrados de modulación y demodulación FSK (XR2211 y XR2206) utilizados para tal fin. Esta condición puede ser susceptible de cambio de acuerdo con las condiciones existentes en un instante dado en la red eléctrica.

- Fácil instalación: cada una de las salidas presenta funciones tales como interruptor, interruptor programado, DIMMER para control de tensión aplicada a la carga.

- El software de control permite obtener una visualización de toda la vivienda controlada, indicando zonas donde hay y no hay energía, dónde hay instalados sensores y su condición (activados o desactivados), aires acondicionados, tomas controladas, y otros elementos domóticos, lo que facilita el control de actividades.

\section{Etapa de diseño del módulo domótico}

A continuación se ilustran los diagramas de bloques que describen cada una de las etapas que hacen parte del desarrollo del proyecto.

Figura 2. Diagrama del esquema de Control

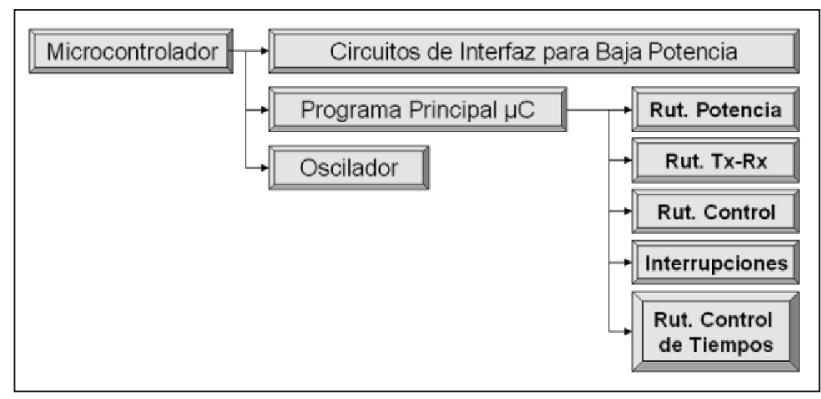

Fuente: el autor

Figura 3. Diagrama de la herramienta Software de Control Domótico

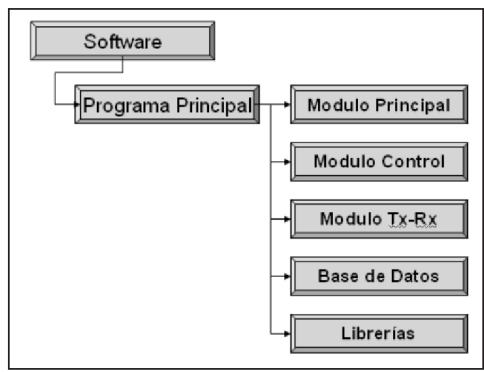

Fuente: el autor 
Figura 4. Diagrama etapa de Potencia



Fuente: el autor

Figura 5. Diagrama sistema de comunicaciones

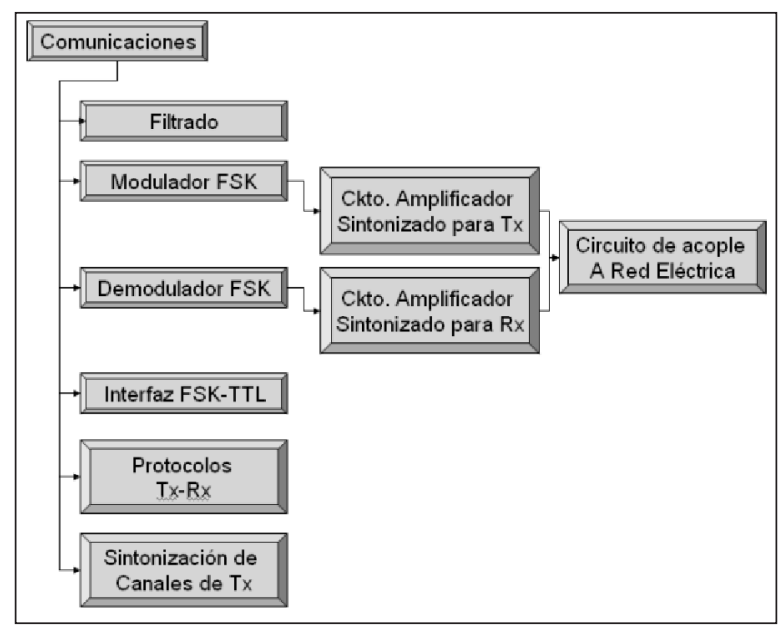

Fuente: el autor

\section{Etapa de comunicación entre el módulo de control y el computador personal pc}

La comunicación serial, como su nombre lo indica, realiza la transferencia de información enviando o recibiendo datos descompuestos en bits, los cuales viajan secuencialmente uno tras otro. Dicha comunicación está compuesta principalmente de dos elementos básicos, el hardware, que hace referencia a la configuración de los conectores y niveles de voltaje, y el software, con el que se controla la información binaria que se quiere transferir. Todo esto está regido por normas o protocolos donde el utilizado por las computadoras convencionales es el Protocolo RS-232.

En el entorno de los microcontroladores es posible establecer la comunicación serial e incluso hay algunos que poseen funciones y registros especiales para ello, tales como la familia $8 \mathrm{X} 5 \mathrm{X}$ de Intel o PIC16F87X de Microchip, que se encargan de manejar todos los aspectos relacionados con las comunicaciones asíncronas, si previamente se han definido sus parámetros. Aún, si un microcontrolador no posee implementada la opción de las comunicaciones seriales, como es el caso del PIC16F84, ésta se puede implementar conociendo el proceso de transmisión serial asíncrono, en cuyo caso es necesario calcular los tiempos de retardo de 1 y 1.5 bits para la construcción de las rutinas de transmisión y recepción correspondientes. 
En el caso particular para establecer una velocidad de transmisión de 600 baudios, es decir, 600 bits por segundo; el tiempo requerido para 1 bit y 1.5 bits es:

Tiempo de $1 \mathrm{Bit}=\frac{1}{600}=1.66^{*} 10^{-3} \mathrm{seg}=1.66 \mathrm{~ms}$

Tiempo de $1.5 \mathrm{Bit}=1.5 * 1.66 \mathrm{~ms}=2.5 \mathrm{~ms}$

Como la frecuencia del cristal seleccionado es de $4 \mathrm{MHz}$, el tiempo que tarda en ejecución 1 ciclo de máquina es de $1 \mu \mathrm{s}$. Este valor se calcula de la siguiente forma:

1 Ciclo de máquina $=4$ ciclos de reloj $=4$ periodos de frec. oscilador

1 Ciclo de máquina $=\frac{4}{4 M H z}=\frac{4}{4 * 10^{6}}=1 \mu \mathrm{s}$

Por lo anterior fue necesario generar retardos de $1.66 \mathrm{~ms}$ y $2.5 \mathrm{~ms}$, implementando un procedimiento de espera utilizando el tiempo correspondiente a 10 ciclos de máquina, proceso que se debe repetir un número determinado de veces según el retardo deseado.

$1.66 \mathrm{~ms}=1 \mu \mathrm{s} * 10 * 166 ; 10$ ciclos de máquina, 166 veces

$2.5 \mathrm{~ms}=1 \mu \mathrm{s} * 10 * 250 \quad ; 10$ ciclos de máquina, 250 veces

El resultado de la rutina que permite generar los retardos de un bit y 1.5 bits, requeridos en cada una de las rutinas de transmisión y recepción serial asincrónica a una velocidad de 600 baudios es la siguiente: (en el recuadro se resalta los ciclos de máquina necesarios para el retardo).

Tabla 1. Ciclos de máquina necesarios para el retardo

\begin{tabular}{|lll|}
\hline UNOYMED & MOVLW .250 & ; Retardo a 1.5 bits \\
UN_BIT & GOTO STARTUP & \\
STARTUP & MOVLW .166 & ; Retardo a 1 bit \\
REDO & NOVWF RETARD & \\
& NOP & ; Duración : 1 ciclo de Maq. \\
& NOP & ; Duración : 1 ciclo de Maq. \\
& NOP & ; Duración : 1 ciclo de Maq. \\
& NOP & ; Duración : 1 ciclo de Maq. \\
& NOP & ; Duración : 1 ciclo de Maq. \\
& NOP & ; Duración : 1 ciclo de Maq. \\
& DECFSZ RETARD & ; Duración : 1 ciclo de Maq. \\
& GOTO $\quad$ REDO & ; Duración : 1 ciclo de Maq. \\
& RETURN & Duración : 2 ciclos de Maq \\
\hline
\end{tabular}


Por otro lado, cada una de las tramas enviadas en el proceso de comunicación está constituida por 8 bits, en donde, los cuatro bits menos significativos corresponden al número de identificación del módulo (numerando los módulos de 1 a 15 , el 0 no se utiliza por ser valor neutro en casos como cambio ángulo de disparo) y los cuatro bits más significativos corresponden a la orden que se desea ejecutar tal como se muestra en la figura.

Figura 6. Protocolo de Comunicación

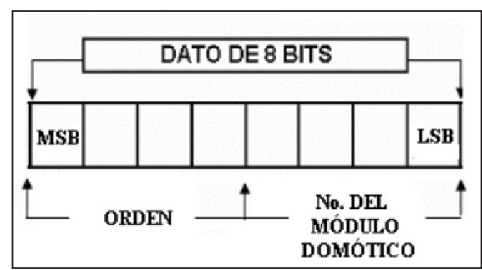

Fuente: el autor

Cuadro 1. Listado de órdenes ejecutadas por el módulo domótico

\begin{tabular}{|c|c|l|}
\hline ORDEN & CÓDIGO & \multicolumn{1}{c|}{ FUNCIÓN } \\
\hline 0 & 0000 & No hace nada "comodin" \\
\hline 1 & 0001 & Dimmer \\
\hline 2 & 0010 & Verificar estado del sensor (activo o no) \\
\hline 3 & 0011 & On_off \\
\hline 4 & 0100 & Cambiar configuración interruptor - dimmer \\
\hline 5 & 0101 & cambiar el número del módulo en eeprom \\
\hline 6 & 0110 & Leer el nÚmero del mÓdulo \\
\hline 7 & 0111 & Verificar estado de la comunicación \\
\hline 8 & 1000 & leer configuración del módulo \\
\hline 9 & 1001 & habilitar y deshabilitar salidas \\
\hline
\end{tabular}

Fuente: el autor

Cuando la función que se desea ejecutar es la función Dimmer, es necesario enviar una trama adicional de ocho bits distribuidos de la siguiente forma:

Figura 7. Trama adicional enviada al módulo domótico cuando se desea establecer un nuevo ángulo de disparo

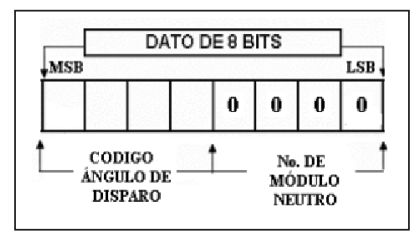

Fuente: el autor 
Cuadro 2. Listado de códigos según el ángulo de disparo deseado

\begin{tabular}{|c|c|}
\hline CÓDIGO & $\begin{array}{c}\text { ÁNGULO } \\
\text { DISPARO }\end{array}$ \\
\hline 0000 & 0 \\
\hline 0001 & 12 \\
\hline 0010 & 24 \\
\hline 0011 & 36 \\
\hline 0100 & 48 \\
\hline 0101 & 60 \\
\hline 0110 & 72 \\
\hline 0111 & 84 \\
\hline 1000 & 96 \\
\hline 1001 & 108 \\
\hline 1010 & 120 \\
\hline 1011 & 132 \\
\hline 1100 & 144 \\
\hline 1101 & 156 \\
\hline 1110 & 168 \\
\hline 1111 & 180 \\
\hline
\end{tabular}

Fuente: el autor

Los cuatro bits menos significativos tendrán valor cero, equivalente al número de identificación neutro del módulo domótico. Los cuatro bits más significativos tomarán valores entre 0000 y 1111 , haciendo referencia al ángulo de disparo deseado según la figura en el cuadro anterior.

La comunicación con el PC es serial asíncrona, el protocolo de comunicación es RS-232/ FSK, transmitiendo a 600 baudios, 8 bits de datos, sin paridad, y con un bit de parada. Como se requiere de una interfase que permita convertir los niveles RS-232 a señales FSK. En el proyecto se utilizó el MAX232 como un integrado que permite convertir los niveles RS-232 a niveles TTL, y los circuitos integrados XR2206 y XR2211, que permiten la modulación y demodulación de señales TTL-FSK.

Figura 8. Interfase de comunicación RS232/FSK

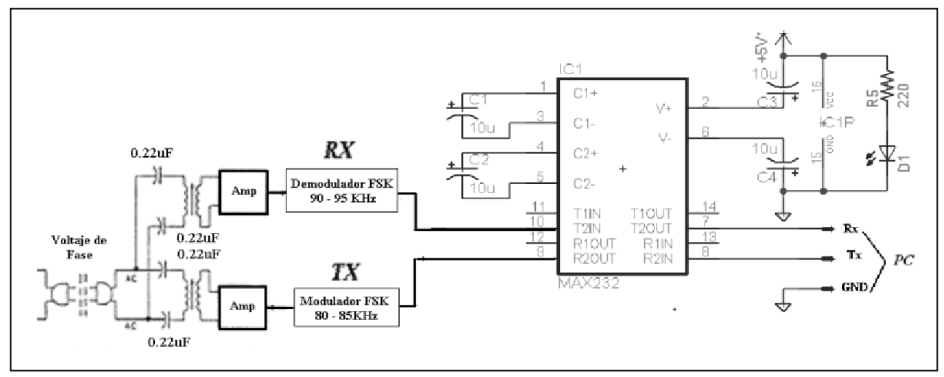

Fuente: el autor 
Para el caso de la aplicación correspondiente a la comunicación serial con el computador personal, se establece la comunicación serial entre el microcontrolador y el PC, empleando las rutinas de transmisión y recepción mediante Visual Basic. Para la utilización del puerto serial en Visual Basic se debe utilizar un control ActiveX llamado MICROSOFT COMM CONTROL, el cual permite manipular las comunicaciones seriales desde cualquier puerto serial existente en el PC y a velocidades configurables por el usuario.

\section{ETAPA DE DE PRUEBA Y CONVALIDACIÓN DE RESULTADOS}

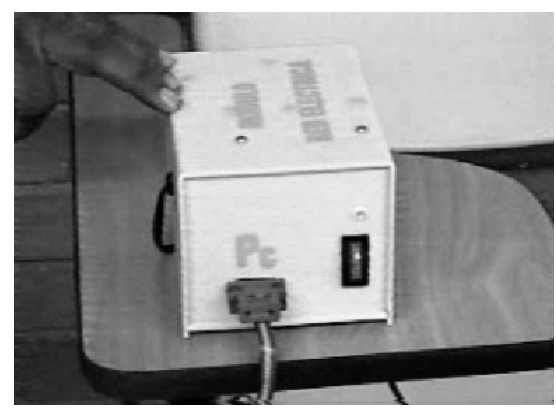

Figura 9. Aspecto Físico del módulo domótico Fuente: el autor

En el momento de efectuar las pruebas de comunicación, la velocidad máxima para comunicación sin pérdida de información fue a 600 baudios, logrando con ello:

- Superar 10 veces la velocidad de comunicación del sistema X10, la cual es de 60 baudios.

- Permitir comunicación semi-duplex

- Envío de datos sin redundancia, es decir, no hay necesidad de enviar varias veces la misma trama de comunicación, tan solo se envía una vez.

- Si el sistema se desea implementar en una red trifásica, será necesario conectar un transmisor y un receptor por fase, debido a que el sistema solo funcionará si el transmisor y el receptor pertenecen al mismo circuito (Sistema Monofásico).

Figura 10. Onda correspondiente al valor 85 (01010101) recibida en PIC16F84 a través del pin RA0 a 600 baudios

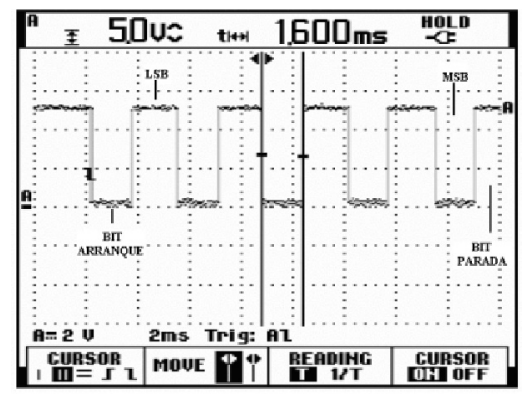

Fuente: el autor 
En la figura anterior, se muestra la forma de onda recibida en PIC16F84 a través del pin RA0, cuando desde el PC se envía serialmente el número 85 (01010101) a una velocidad de 600 baudios, 8 bits de datos, un bit de parada y sin paridad.

Considerando que:

El tiempo de 1 Bit $=\frac{1}{600}=1.66 * 10^{-3} \mathrm{seg}=1.66 \mathrm{~ms}$.

\section{Descripción del funcionamiento del software}

El software fue diseñado en Visual Basic 6.0 y es el encargado de realizar el control domótico. Está constituido por dos formularios, uno de ellos es el formulario principal en el cual se puede construir el plano de la vivienda por plantas o pisos, con una capacidad máxima de 50 pisos. El otro formulario es el formulario de configuración de los módulos domóticos, en el cual se pueden programar las diferentes funciones de los módulos tales como horarios de funcionamiento, Dimmer, estado de los interruptores, verificación de la comunicación, habilitación de salidas de potencia y configuración de numeración del módulo. Este formulario sufre cambios o modificaciones en su apariencia según el tipo de módulo que se está trabajando.

Figura 11. Formulario principal módulo domótico

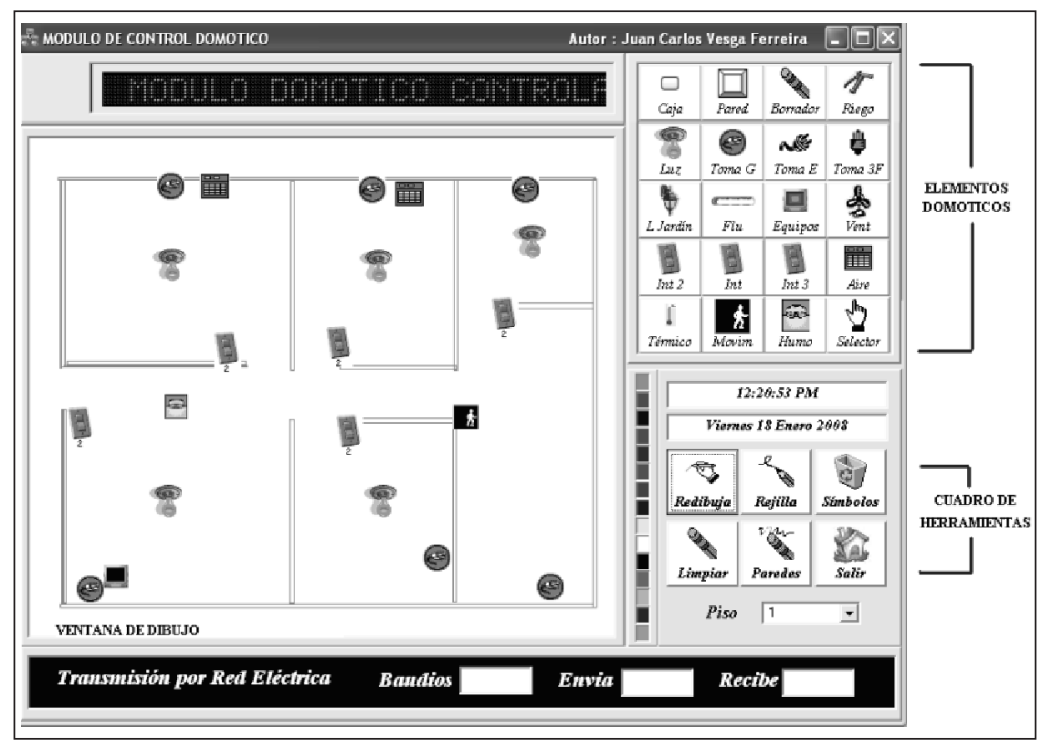

Fuente: el autor

Elementos domóticos: en este cuadro se encuentran todos los elementos necesarios para la configuración del sistema domótico. Es la zona donde encontramos aquellos elementos que nos permiten dibujar el plano residencial, ilustrando paredes, lámparas incandescentes, toma 
corrientes, electrodomésticos, aires acondicionados, sensores de movimiento, sensores de humo, sensores de temperatura, interruptores sencillos, interruptores dobles e interruptores triples, los cuales son equivalentes a los módulos domóticos, indicando cuantas salidas desean controlar como máximo. Por ejemplo, si se dibuja un interruptor doble significa que el módulo al cual representa, de las tres salidas que posee solamente utilizará dos y siempre se encontrará una salida deshabilitada.

Figura 12. Cuadro de elementos

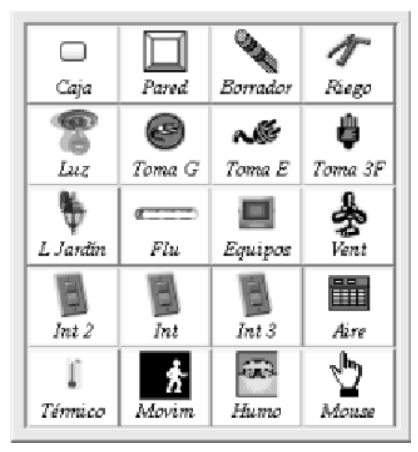

Fuente: el autor

El elemento domótico seleccionado por es usuario se ubica en la ventana de dibujo (plano residencial) haciendo clic con el botón izquierdo del mouse en el punto donde se desea dibujar, en donde se configura el nombre del elemento, el número de identificación del módulo que lo controla y la salida de potencia del módulo sobre el cual actúa.

Cuando se ha diseñado el plano residencial y se han ubicado los elementos deseados en las zonas requeridas, pueden ser configurados cada uno de ellos, escogiéndose el elemento MOUSE, ubicado en el cuadro de elementos y seleccionando el elemento a configurar.

Figura 13. Formulario de configuración de elementos

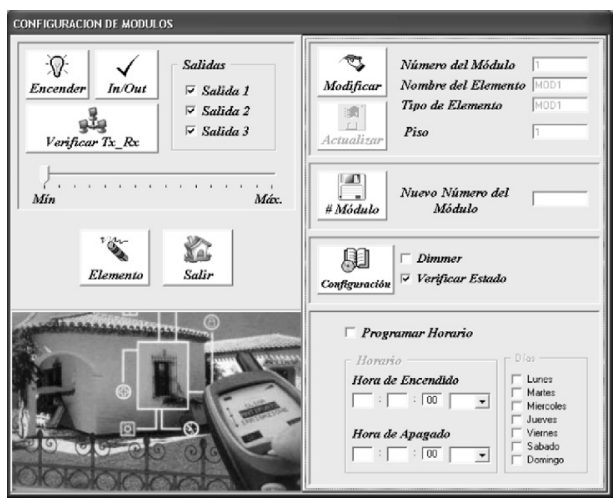

Fuente: el autor 
Para comprender mejor la forma como se configugura un módulo domótico, se ilustrará a continuación cada una de las ventanas que componen este formulario.

Figura 14. Información general del módulo

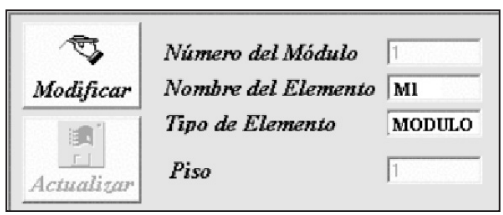

Fuente: el autor

En la figura anterior se muestra la información básica del módulo como el nombre del elemento, número del módulo, tipo de elemento y piso en el que se encuentra. Cuando se pulsa la opción modificar, permite solamente cambiar la información correspondiente al nombre del módulo y el piso en el que se encuentra.

Figura 15. Cambio de número de identificación del Módulo

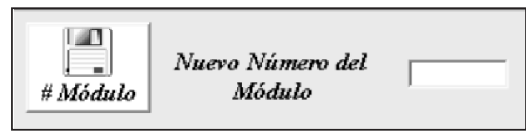

Fuente: el autor

Si se desea cambiar el número que identifica al módulo domótico, se debe ubicar el usuario en la ventana correspondiente a la figura anterior, en la cual se escribe el nuevo número de identificación del módulo, seguido de "enter" o hacer clic en el botón “\#Módulo".

Figura 16. Ventana de configuración de función Dimmer y monitoreo de sensores

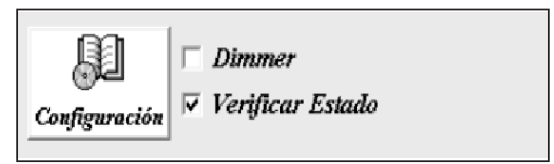

Fuente: el autor

La ventana mostrada en la figura, permite habilitar y deshabilitar las funciones de Dimmer y detección de estado del sensor (Activado o Desactivado) conectado al módulo domótico.

Cuando se habilita la opción Dimmer, el control de tensión suministrado a la carga (Min = $0 \%$ de tensión, Máx. $=100 \%$ de tensión), se debe efectuar utilizando el deslizador que se muestra en la figura siguiente. 
Figura 17. Deslizador para control de tensión suministrada a la carga

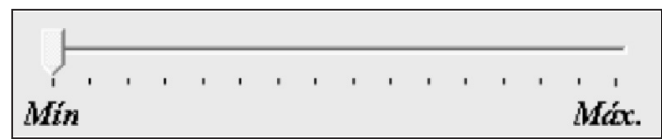

Fuente: el autor

Cuando se habilita la función "Verificar Estado", se activa en el módulo el monitoreo del sensor. Por ejemplo, supongamos que se trata de un sensor de movimiento, el cual posee internamente un relé que en condiciones de no detección de intrusos, mantiene su estado en OFF. Cuando el sensor detecta movimiento en el recinto, el relé interno cambia de estado, reflejando este cambio en el estado de sus contactos, provocando un cambio de nivel de tensión en el módulo domótico, el cual a su vez le indica al PC este cambio y el software activa una señal sonora describiendo este evento. Para el caso, la reproducción de un mensaje "iAlerta!, Se ha detectado la presencia de un intruso" además de una señal visual, cambiando de color el elemento que representa al sensor activado.

Figura 18. Ventana para habilitación de salidas



Fuente: el autor

En la figura anterior, se muestra la ventana que permite habilitar y deshabilitar las salidas controladas por los triacs que gobiernan la parte de potencia.

Figura 19. Ventana de programación de horario ON/OFF

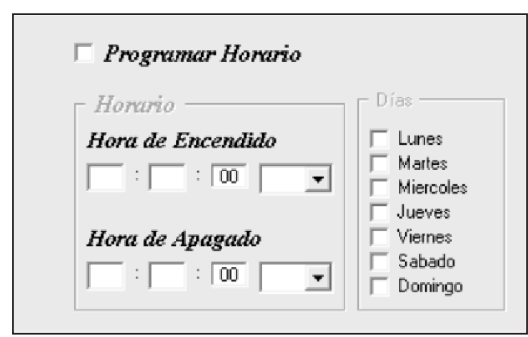

Fuente: el autor

En la figura anterior se muestra la ventana de configuración de horario On/Off de las salidas del módulo domótico, indicando la hora de encendido, la hora de apagado y la selección 
de los días en que se utilizará este horario. Se debe tener en cuenta que para habilitar esta función, hay que seleccionar la opción "Programar horario".

\section{CONCLUSIONES}

PLC surge como una alternativa tecnológica viable económicamente, la cual hace uso de la red eléctrica domiciliaria, brindando la posibilidad de implementar una red de área local a bajo costo y de utilizar infraestructura de cableado ya existente, lo cual es una gran ventaja frente a otras tecnologías, y que permite llegar a diferentes sitios donde otros medios de transmisión no llegarían tan fácilmente.

La tecnología PLC posibilita la transmisión de información a través de los cables eléctricos de baja tensión que llegan a los hogares, convirtiendo cualquier toma de corriente existente en la casa, en una conexión a todos los servicios de telecomunicación.

El creciente interés de la tecnología PLC en Colombia, desde el punto de vista académico y empresarial se ha ido consolidando a partir de los últimos años, puesto que se ha contado con el apoyo del Estado y la empresa privada.

En el mundo moderno, con el desarrollo de la Domótica (automatización residencial) y los edificios inteligentes, la tecnología PLC permite proveer un medio de comunicación entre diversos dispositivos existentes en el hogar como por ejemplo: sensores, alarmas, electrodomésticos, interruptores entre otros.

Actualmente, los avances en la tecnología PLC en Europa y Estados Unidos permiten alcanzar altas velocidades de transmisión y mayor ancho de banda sobre líneas de bajo y medio voltaje, lo cual facilita la transformación de la red eléctrica en una auténtica red de banda ancha, capaz de soportar servicios de datos, voz y vídeo, además de cualquier otro servicio que ofrezca un operador de telecomunicaciones.

"Fabricantes como Toyocom (www.toyocom.co.jp/english/) y Corinex (www.corinex. com) disponen ya de productos comerciales que utilizan la nueva generación de la tecnología PLC capaces de alcanzar velocidades de hasta $200 \mathrm{Mbps}$, incorporados en los últimos diseños desarrollados por DS2 (tecnología Wisconsin)” (Teconocom, 2007).

Se desarrolló un sistema de carácter didáctico que permite emular una instalación domótica en la cual se puede conceptualizar las funciones y ventajas que estos sistemas brindan al usuario. Este sistema Inteligente asistido por computador permite el control, supervisión y programación de diferentes módulos domóticos presentes en el plano residencial.

Se desarrolló un módulo domótico basado en el microcontrolador PIC16F84, el cual se escogió por varias razones: la primera por tener el número de pines configurables como entrada/salida requeridos para el diseño. El segundo parámetro de selección fue la memoria EEPROM, debido a que la concepción del diseño didáctico requería cambiar la identificación del módulo por medio del software cada vez que se desee. Este módulo se puede programar 
de manera independiente a través del software de control según su identificación, lo que permite conformar sistemas de control a gran escala en forma modular.

El acople con la red eléctrica se efectuó utilizando un transformador de frecuencia intermedia referencia 22306 amarillo, el cual posee un valor de inductancia de $0.1 \mathrm{mH}$. Este transformador fue acoplado capacitivamente con dos condensadores de valor $0.22 \mu \mathrm{F}$, con los cuales se obtuvo la mejor respuesta para las frecuencias comprendidas entre $85 \mathrm{KHz}$ y $95 \mathrm{KHz}$.

En el momento de efectuar las pruebas de comunicación, la velocidad máxima para comunicación en el sistema de red eléctrica sin pérdida de información fue a 600 baudios, logrando con ello:

- Superar 10 veces la velocidad de comunicación del sistema X10, la cual es de 60 baudios.

- Permitir comunicación semi-duplex

- Envío de datos sin redundancia, es decir, no hay necesidad de enviar varias veces la misma trama de comunicación, tan solo se envía una vez.

- El sistema está diseñado para red monofásica

La comunicación se realizó empleando un sistema similar al maestro-esclavo, donde el PC hace las veces de maestro y es el encargado de enviar las órdenes y la configuración de cada uno de los módulos domóticos conectados a la red. El módulo, que hace las veces de esclavo, retransmite una trama (dirección + función) que recibió como orden, con lo cual el controlador o maestro compara bit a bit la trama recibida con la enviada confirmando que el módulo domótico recibió la orden correctamente.

Debido a que los datos a transmitir no son de gran extensión, se optó por utilizar un protocolo de comunicación propio, que permite configurar todos los equipos que se vayan a conectar a la red. El sistema no detecta la presencia de equipo conectado a la red sin configuración previa.

El software desarrollado en Visual Basic 6.0 que es el encargado de realizar el control domótico, está constituido por dos formularios, uno de ellos es el formulario principal en el cual se puede construir el plano de la vivienda por plantas o pisos, con una capacidad máxima de 50 pisos. El otro formulario es el de configuración de los módulos domóticos, en el cual se pueden programar las diferentes funciones de los módulos tales como horarios de funcionamiento, Dimmer, estado de los interruptores, verificación de la comunicación, habilitación de salidas de potencia y configuración de numeración del módulo.

Se logró variar el ángulo de disparo de los Triacs grado a grado y de manera precisa, gracias a la exactitud que se pudo alcanzar con el microcontrolador, debido a que este nos brinda las ventajas de trabajar con un cristal de cuarzo y no con una red de tipo RC con la cual se hace convencionalmente.

La domótica se muestra como toda una promesa que revolucionará muchos de los hábitos del hombre, facilitando las actividades mecánicas, monótonas o triviales brindando mayor 
seguridad dentro del hogar. Por el momento, los altos costos de instalación de estos sistemas, hacen que la domótica llegue a un número limitado de usuarios; sin embargo, la tecnología seguirá su natural proceso exponencial de desarrollo, ofreciendo en un par de años más el acceso a estos sistemas integrales de control casero apta para toda la familia.

\section{REFERENCIAS BIBLIOGRÁFICAS}

BAIG, S., GOHAR, N. D. (2003), “A discrete multitone transceiver at the heart of the PHY layer of an In-Home Powerline Communication Local-Area Network," IEEE Commn. Mag., Vol. 41, n. o. 4, págs. 48-53, Apr 2003.

BROWN PA, "Power line communications - past, present, and future," Proceedings of Internacional Symposium on Power-line Communications and its Applications, September 1999, 1-8.

CHECA, Luís María. (2000), Líneas de transporte de energía tercera edición. Editorial Alfaomega Octubre de 2000.

GARCÍA, Jesús. (2006), “Redes para proceso distribuido”. 3. a Ed. Alfaomega.

GUTIÉRREZ P., Humberto, “Análisis y diseño de experimentos”, 2 Ed. McGraw-Hill 2008.

KAIZAWA Y, MARUBAYASHI G., "Needs for the power line communications," Proceedings of Internacional Symposium on Power-line Communications and its Applications, 1998, 153-157.

LAMAS, Javier. (1998), Sistemas de control para viviendas y edificios. 1. a ed., México, Ed. Paraninfo.

LEE, J., TRIPATHI, K. (2007), "Efficient High Speed Communications over Electrical Powerlines for a Large Number of Users", Scientific and technical Publishing, 2007.

MARTÍNEZ B., Ciro, (1998), “Estadística y Muestreo”, 9.a Ed. Ecoe ediciones.

SANDOVAL, Juan. (1999), Domótica. 1. a ed. México, Ed. Paraninfo. ISBN 84-2832819-5

STALLINGS, William, (2000), Comunicaciones y redes de computadores. 6. a ed. Madrid, España: Pearson Educación. 